\title{
Healthcare resources and expenditure in financial crisis: scenarios and managerial strategies
}

\author{
Sabina Nuti, Milena Vainieri \& Marco Frey \\ Institue of Management - Sant'Anna School of Advanced Studies of Pisa - piazza Martiri della Libertà, 27 - 56127 Pisa, Italy
}

\begin{abstract}
Objective: What are the implications of financial crisis on healthcare expenditure? This paper explores different approaches applied across European countries focusing on the role that managerial tools may have in coping with this challenge. Method: The paper reports the results of recent studies on responses to financial crisis from European countries and which are the techniques they had applied to reallocate resources. Results: Although resources scarcity, some governments did not reduce the healthcare expenditure because they believe in its focal role on the economic development and on maintaining social cohesion and protection of vulnerable people. Other countries decided a strong reduction of costs which often has affected services delivered. In both cases authors suggest to avoid across-the-board cuts in favor of approach involving priority setting. Conclusion: The public sector has assumed new responsibilities following the global crisis and the rising demand for social services. Some countries shifted the healthcare costs from the public purse to private households undermining the survival of the health system and the universal coverage. A way to avoid this risk is based on the ability to share discussion about where to cut and where to reallocate resources.
\end{abstract}

Keywords: Healthcare resources, variability, financial crisis, reallocation 